\title{
ВMJ Global Health Effectiveness of intermittent screening and treatment for the control of malaria in pregnancy: a cluster randomised trial in India
}

To cite: Kuepfer I, Mishra N, Bruce J, et al. Effectiveness of intermittent screening and treatment for the control of malaria in pregnancy: a cluster randomised trial in India. BMJ Global Health 2019;4:e001399. doi:10.1136/ bmjgh-2019-001399

Handling editor Soumitra Sudip Bhuyan

- Additional material is published online only. To view, please visit the journal online (http://dx.doi.org/10.1136/ bmjgh-2019-001399).

Received 14 January 2019 Revised 16 May 2019 Accepted 25 May 2019

Check for updates

(c) Author(s) (or their employer(s)) 2019. Re-use permitted under CC BY-NC. No commercial re-use. See rights and permissions. Published by BMJ.

For numbered affiliations see end of article.

Correspondence to Dr Irene Kuepfer; irene.kuepfer@Ishtm.ac.uk

\section{ABSTRACT}

Background The control of malaria in pregnancy (MiP) in India relies on testing women who present with symptoms or signs suggestive of malaria. We hypothesised that intermittent screening and treatment for malaria at each antenatal care visit (ISTp) would improve on this approach and reduce the adverse effects of MiP.

Methods A cluster randomised controlled trial comparing ISTp versus passive case detection (PCD) was conducted in Jharkhand state. Pregnant women of all parities with a gestational age of 18-28 weeks were enrolled. Women in the ISTp group were screened with a rapid diagnostic test (RDT) for malaria at each antenatal clinic visit and those in the PCD group were screened only if they had symptoms or signs suggestive of malaria. All RDT positive women were treated with artesunate/sulfadoxine-pyrimethamine. The primary endpoint was placental malaria, determined by placental histology, and the key secondary endpoints were birth weight, gestational age, vital status of the newborn baby and maternal anaemia.

Results Between April 2012 and September 2015, 6868 women were enrolled; 3300 in 46 ISTp clusters and 3568 in 41 PCD clusters. In the ISTp arm, $4.9 \%$ of women were tested malaria positive and $0.6 \%$ in the PCD arm. There was no difference in the prevalence of placental malaria in the ISTp $(87 / 1454,6.0 \%)$ and PCD $(65 / 1560,4.2 \%)$ groups (6.0\% vs $4.2 \%$; OR $1.34,95 \% \mathrm{Cl} 0.78$ to $2.29, \mathrm{p}=0.29$ ) or in any of the secondary endpoints.

Conclusion ISTp detected more infections than PCD, but monthly ISTp with the current generation of RDT is unlikely to reduce placental malaria or impact on pregnancy outcomes. ISTp trials with more sensitive point-of-care diagnostic tests are needed.

\section{INTRODUCTION}

Malaria in pregnancy (MiP) is a major public health problem, contributing substantially to morbidity and mortality among pregnant women, developing fetuses and newborn babies in endemic areas. ${ }^{12}$ For the control of

\section{Key questions}

What is already known?

- In low malaria transmission areas, testing pregnant women for malaria when they present with clinica symptoms (passive case detection (PCD) is the standard of care.

- In high malaria transmission areas, intermittent preventive treatment with sulfadoxine-pyrimethamine (IPT-SP) during monthly antenatal visits is the standard of care to reduce the consequences of malaria in pregnancy (MiP).

- It has been shown that intermittent screening with a rapid diagnostic test and treatment for malaria (ISTp) during antenatal visits is not inferior to IPT-SP.

What are the new findings?

- In a low transmission area in India, $70 \%$ of MiP was asymptomatic and ISTp detected more cases of MiP than PCD $(4.9 \%$ vs $0.6 \%, p<0.001)$, but had no impact on the risk of placental malaria or other adverse birth outcomes.

What do the new findings imply?

- Given that the majority of women infected with malaria remain asymptomatic, ISTp during antenatal care could be considered in low transmission areas in India.

MiP, the WHO recommends effective treatment, insecticide-treated bed nets (ITNs) and, in areas of moderate-to-high malaria transmission of sub-Saharan Africa, intermittent preventive treatment with sulfadoxinepyrimethamine (IPTp-SP) ${ }^{3}$ IPTp-SP is given at each antenatal clinic (ANC) attendance after the first trimester, regardless of whether the recipient is infected with malaria or not. In low transmission settings, the use of IPTp-SP is questionable because the benefit/risk ratio of this intervention is likely to be low. ${ }^{4}$ 
In such areas, intermittent screening for malaria during antenatal visits using a rapid diagnostic test (RDT) and treating test-positive women with an effective antimalarial drug (ISTp) is a potential alternative option. ${ }^{56}$

In India, malaria transmission is relatively low and varies markedly between different ecological settings. ${ }^{7}$ A study conducted in the eastern state of Jharkhand in 2007 showed that placental parasitaemia, measured by RDT or placental smear microscopy, ranged from $0.8 \%$ in urban areas to $3.6 \%$ in rural areas. However, it is estimated that 28.2 million pregnancies are at risk of Plasmodium falciparum $(P f)$ malaria annually in India, and thus even a low prevalence can translate into substantial mortality and morbidity. ${ }^{8}$ Especially pregnant women from tribal populations and residents of forested hilly areas are known to be at higher risk of infection. ${ }^{9}$ The current national policy in India for the control of MiP is to test pregnant women who present with symptoms or signs suggestive of malaria with a blood slide and to treat confirmed cases using quinine in the first trimester and artesunate (AS)+SP or artemether+lumefantrine in the second or third trimester. The use of a long-lasting insecticide-treated net (LLIN) during pregnancy is recommended in high-risk areas, but there is no specific distribution system for LLINs linked with antenatal care services. The effectiveness of the current policy of passive case detection (PCD) of MiP in low transmission settings is unknown. We hypothesised that ISTp would be more effective than PCD in detecting MiP and that effective treatment of infected individuals would reduce the risk of placental malaria and adverse birth outcomes. In this paper, we report a study that evaluated the effectiveness of ISTp delivered through the routine antenatal care system in a moderate-to-low transmission setting in India.

\section{METHODS}

\section{Study design}

This was a two-arm, cluster randomised controlled trial that compared ISTp versus the current policy of PCD for malaria during antenatal care visits. The primary endpoint of the trial was placental malaria and the secondary endpoints were low birth weight, adverse fetal birth outcomes and maternal anaemia. The unit of randomisation was sub-health centres (SHC). Women were assigned to the ISTp arm or the PCD arm depending on the allocation of the SHC, where they attended ANC.

\section{Study area and population}

The study was conducted in Jharkhand state, India, in an area surrounded by forest, where malaria transmission peaks during the rainy season from June to October. The study was implemented within the primary healthcare delivery system in four subdistricts, two from Gumla district (Kamdara and Basia) and two from Simdega district (Bano and Kolebira). The population in each of the four subdistricts ranged from 63775 to $80731 .{ }^{10}$ Each subdistrict has one primary health centre (PHC) and several SHCs, those have at least one auxiliary midwife (ANM) who provides antenatal care. In Basia subdistrict, there is a government hospital and the St. Ursula mission hospital, which served as the study hospital. Delivery services are available in all PHCs and in approximately $75 \%$ of SHCs. Emergency obstetric care, including caesarean section, is provided in the government and mission hospital in Basia.

\section{Sample size and randomisation}

During an observational study done in 2007, $4 \%$ of women attending ANC at St. Ursula mission hospital had peripheral malaria parasitaemia. ${ }^{11}$ Assuming the risk of placental malaria would be higher than that of peripheral parasitaemia, we expected that $7.5 \%$ of women in the PCD arm would have placental malaria, and we hypothesised that ISTp would reduce the risk of placental malaria by $25 \%$. In order to detect a $25 \%$ reduction in the risk of placental malaria with $80 \%$ power, a two-sided alpha of 0.05 and a design effect of 1.25 , the study needed 31 clusters per arm and 100 women per cluster, total 6200 women. Women attending an individual SHC for antenatal care comprised a cluster. As there were 80 SHCs in the four study subdistricts and it was uncertain whether it would be possible to enrol 100 women per cluster in 2 years, we randomised all 80 SHCs (40:40). We stratified the SHCs by subdistricts and then assigned numbers to randomise them to two groups.

As the enrolment rate was slower than expected, and eight SHCs had to be closed due to security concerns, we replaced them with 15 maternal and children care centres (Anganwadi centres) in the subdistrict of Bano in July 2012.

\section{Trial procedures}

All staff who provided antenatal and delivery care in the study SHCs, PHCs and hospital, and the project field staff were trained to follow trial standard operating procedures and to complete study case record forms. All eligible pregnant women attending at an ANC at the study SHCs were enrolled in the study after informed consent had been obtained. Pregnant women of all parities with a gestational age of 18-28 weeks were eligible for enrolment. Pregnant women with a chronic illness were excluded. All study participants were given free health and delivery services at the study hospital, PHCs or SHCs.

At enrolment, women in both groups received routine antenatal care. A finger prick blood sample was taken for the assessment of haemoglobin (HemoCue, Angelholm, Sweden) and for the collection of a filter paper sample. Syphilis and HIV testing were only done if part of routine ANC. In the ISTp group, all women had an RDT (SD Bioline Malaria Ag Pf/Pv (histidine-rich-protein 2 (HRP-2)/plasmodium lactate dehydrogenase (pLDH), www.Standardia.com)), a blood smear and filter paper sample (Whatman Grade $3 \mathrm{Chr}$ ) collected, irrespective of a history of fever, at each ANC attendance. In the PCD group, only women with a history of fever within 
Table 1 Comparison of baseline characteristics between the two study groups

\begin{tabular}{|c|c|c|}
\hline & ISTp & PCD \\
\hline & $\mathbf{N}(\%)$ & $\mathbf{N}(\%)$ \\
\hline Number of clusters & 46 & 41 \\
\hline Number of women & 3300 & 3568 \\
\hline \multicolumn{3}{|l|}{ Women per cluster } \\
\hline Median (range) & 93 (6-224) & $92(10-264)$ \\
\hline \multicolumn{3}{|l|}{ Age group, years } \\
\hline$<20$ & $170(5.2)$ & $176(4.9)$ \\
\hline $20-24$ & $1326(40.2)$ & 1405 (39.3) \\
\hline $25-29$ & $1159(35.2)$ & $1241(34.8)$ \\
\hline $30+$ & $644(19.5)$ & $746(21.0)$ \\
\hline
\end{tabular}

Gravidity

$\begin{array}{lrr}\text { Primi- } & 1218(36.9) & 1334(37.4) \\ \text { Secondi- } & 955(28.9) & 973(27.3) \\ \text { Multi- } & 1127(34.1) & 1261(35.4)\end{array}$

Education

\begin{tabular}{|crr|}
\hline None & $977(29.6 \%)$ & $976(27.4)$ \\
\hline Primary & $1128(34.2)$ & $1253(35.1)$ \\
\hline Secondary & $706(21.4)$ & $771(21.7)$ \\
\hline Higher & $488(14.8)$ & $567(15.9)$
\end{tabular}

\begin{tabular}{l}
$\begin{array}{l}\text { Slept under net previous } \\
\text { night }\end{array}$ \\
Yes \\
\hline Sept under an ITN
\end{tabular}

\begin{tabular}{|c|c|c|}
\hline \multicolumn{3}{|c|}{$\begin{array}{l}\text { Slept under an ITN } \\
\text { previous night }\end{array}$} \\
\hline Yes & $338(13.8)$ & $400(13.7)$ \\
\hline \multicolumn{3}{|c|}{ Socioeconomic quintile } \\
\hline Poorest & $670(20.4)$ & 700 (19.7) \\
\hline 2 & $718(21.8)$ & $696(19.6)$ \\
\hline 3 & 669 (20.6) & $657(18.5)$ \\
\hline 4 & $679(20.4)$ & 694 (19.5) \\
\hline Least poor & $556(16.9)$ & $810(22.8)$ \\
\hline \multicolumn{3}{|c|}{ Haemoglobin (g/L) } \\
\hline$<70$ & $34(1.0)$ & $27(0.76)$ \\
\hline 70 to $<110$ & $2800(84.9)$ & $2912(81.8)$ \\
\hline$\geq 110$ & $465(14.1)$ & $623(17.5)$ \\
\hline
\end{tabular}

\begin{tabular}{|c|c|c|}
\hline \multicolumn{3}{|c|}{$\begin{array}{l}\text { History of fever } \\
\text { ( }<48 \text { hours) or } \\
\text { temperature }>37.5^{\circ} \mathrm{C}\end{array}$} \\
\hline Yes & 279 (8.5) & $182(5.1)$ \\
\hline RDT done & $3163(95.8)$ & 108 (3.0) \\
\hline RDT positive & 101 (3.2) & $10(9.3)$ \\
\hline \multicolumn{3}{|l|}{$\begin{array}{l}\text { RDT species } \\
\text { specification }\end{array}$} \\
\hline P. falciparum & $84(2.7)$ & $7(6.5)$ \\
\hline P. vivax & $9(0.3)$ & $2(2.0)$ \\
\hline
\end{tabular}

Continued
Table 1 Continued

\begin{tabular}{lcc}
\hline & \multicolumn{1}{c}{ ISTp } & PCD \\
\cline { 2 - 3 } & N (\%) & N (\%) \\
\hline Mixed infection $(P f+P v)$ & $8(0.3)$ & $1(0.9)$ \\
PCR done & $2620(79.4)$ & $2706(75.8)$ \\
PCR positive & $155(5.9)$ & $114(4.2)$ \\
Symptomatic malaria* & $30(29.7)$ & $10(100.0)$ \\
\hline
\end{tabular}

${ }^{*}$ RDT positive plus history of fever ( $<48$ hours) or temperature $>37.5^{\circ} \mathrm{C}$.

ISTp, intermittent screening and treatment during pregnancy; ITN, insecticide-treated bed net; $P$., Plasmodium; PCD, passive case detection; Pf, Plasmodium falciparum; Pv, Plasmodium vivax; RDT, rapid diagnostic test.

the past 48 hours or an axillary temperature $>37.5^{\circ} \mathrm{C}$ were tested for malaria with an RDT and had a blood slide and filter paper sample collected. Women who had a positive RDT for malaria were treated with AS+SP according to the national treatment guidelines. The treatment was provided by the ANMs but was not supervised. Women in both groups were advised to attend ANCs regularly every month, and to attend a study health facility if they had any illness in between scheduled ANC visits. In both groups, if a woman presented with a history of fever in between scheduled ANC visits, they had an RDT done,

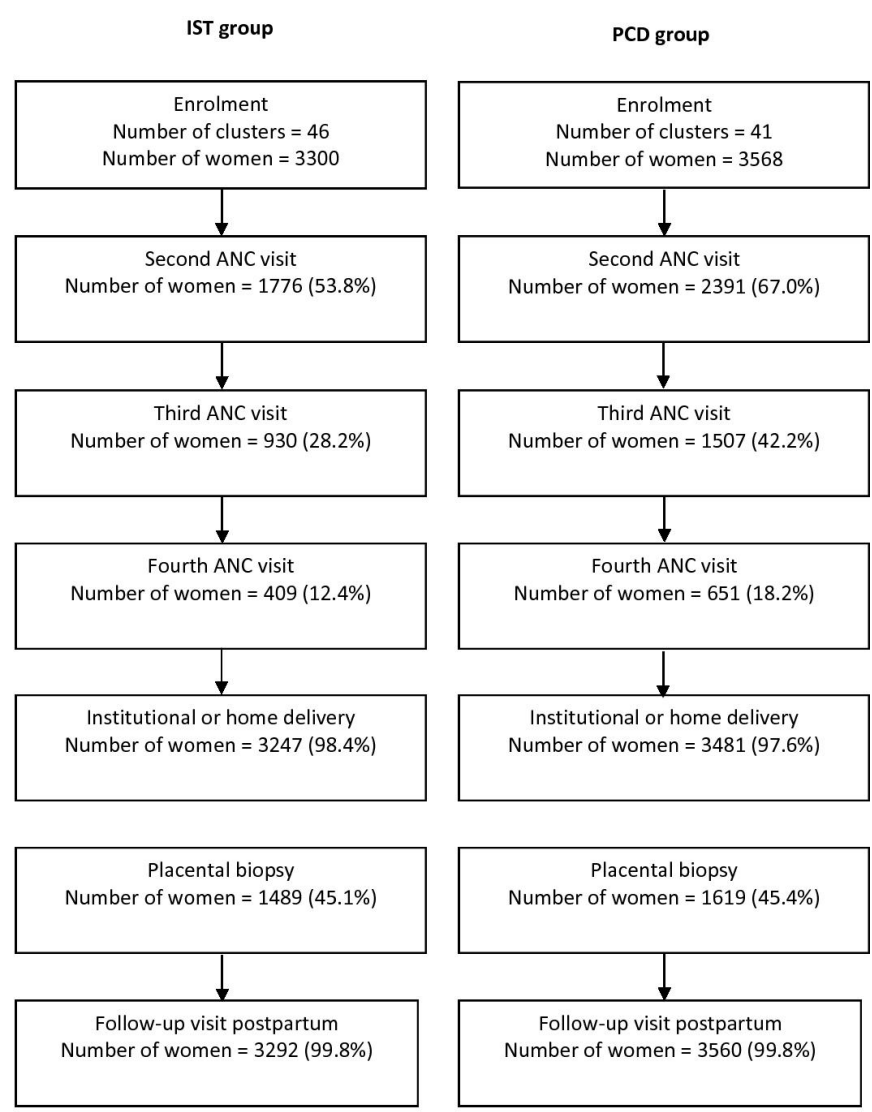

Figure 1 Consort chart. ANC, antenatal clinic; IST, intermittent screening and treatment; PCD, passive case detection. 
blood slide and filter paper sample collected and, if the RDT was positive, they were treated with AS+SP.

At delivery, birth outcomes, birth weight, and the Ballard score of the newborn were assessed, and blood samples for haemoglobin, microscopy and PCR were collected from both groups of women. A placental biopsy and blood smear were taken from all women who delivered in the study hospital or had an institutional delivery within the study area. If a woman delivered at home, or in an institution other than the study health facilities, delivery outcomes were assessed ideally within 7 days of delivery. Study participants were followed once every 2 months up to 6 months postpartum to assess the health status of the mother and her child. A verbal autopsy (VA) was done for all maternal deaths using the WHO 2016 VA questionnaire ${ }^{12}$ and the cause of death was assigned using the InterVA4 algorithm. ${ }^{13}$

\section{Laboratory methods}

Blood slides were fixed and stained with Giemsa. All peripheral and placental blood slides were read twice by study laboratory technicians. For quality control of microscopy, experienced laboratory technicians from the National Institute of Malaria Research, Rourkela Field Station, re-read all slides reported positive by the study laboratory technicians and $50 \%$ of negative slides in year $1,30 \%$ in year 2 and $10 \%$ in year 3 .

Placental biopsies $(2.5 \times 2.5 \times 1 \mathrm{~cm})$ were collected by ANMs at the time of delivery, stored in $10 \%$ buffered formalin containers and taken to the study hospital. On a weekly basis, the biopsies were taken to a private pathology laboratory in Ranchi where they were processed into paraffin tissue blocks. From each block, a set of two slides was prepared, one H\&E and one Giemsa stained. The slides were read by two pathologists (SS and $\mathrm{PB}$ ) based at the Ispat General Hospital, Rourkela, who were trained on-site by a malaria histopathology expert (AM) from the Centers for Disease Control, Atlanta. All histopathological slides were read by both pathologists and slides with discordant results were read again by both pathologists to reach a final diagnosis by consensus. The expert pathologist read $100 \%$ of slides that were classified as placental malaria positive and $30 \%$ of slides classified as negative by the site pathologists. In case of discordant readings, the expert's opinion was deemed to be the final reading. If malaria parasites were seen in the biopsy, it was classified as active placental malaria. Active placental malaria was further classified as acute if there were no malaria pigments in fibrin and chronic if there were malaria pigments in fibrin. If there were no parasites, but there were malaria pigments in fibrin, the sample was classified as past placental malaria. ${ }^{14}$

Filter paper samples taken at enrolment and at delivery (peripheral and placental blood) were tested by PCR in a laboratory in Bangalore, India (Ecron Acunova). DNA extraction was done using the QIAamp Blood DNA Mini Extraction Kit from Qiagen. Real-time (RT) PCR assays, using the Taqman principle, were done with the Genome RT PCR Malaria Kit from Genome Diagnostics.

\section{Data management and analysis}

Data were double entered and verified using the EpiData V.3.1 software (www.epidata.dk). Data processing and analysis were done using the statistical software package STATA V.IC14.0.

All women who were enrolled and for whom information on pregnancy outcomes was available were included in the analysis (intention-to-treat analysis). Principal components analysis was used to obtain a socioeconomic score based on household characteristics and assets. This

Table 2 Prevalence of placental malaria among women who had an institutional delivery within the study area

\begin{tabular}{|c|c|c|c|c|c|}
\hline & \multirow{2}{*}{$\begin{array}{l}\text { IST } \\
\mathrm{N}(\%)\end{array}$} & \multirow{2}{*}{$\begin{array}{l}\text { PCD } \\
\mathbf{N}(\%)\end{array}$} & \multirow{2}{*}{$\begin{array}{l}\text { Adjusted } \\
\text { OR }^{\star}\end{array}$} & \multirow[b]{2}{*}{$95 \% \mathrm{Cl}$} & \multirow[b]{2}{*}{$P$ value } \\
\hline & & & & & \\
\hline Total number of women & 1469 & 1584 & - & & \\
\hline Placental blood RDT done & $1405(95.6)$ & $1540(97.2)$ & - & & \\
\hline \multicolumn{6}{|l|}{ Placental blood RDT result } \\
\hline P. falciparum & $28(2.0)$ & $21(1.4)$ & - & & \\
\hline P. vivax & $2(0.1)$ & $6(0.4)$ & & & \\
\hline Mixed infection $(P f+P v)$ & $2(0.1)$ & $4(0.3)$ & & & \\
\hline Placental histology done & $1454(98.9)$ & $1560(98.5)$ & & & \\
\hline Any placental malaria & $87(6.0)$ & $65(4.2)$ & 1.34 & 0.78 to 2.29 & 0.29 \\
\hline Active infection & $30(2.1)$ & $27(1.7)$ & 1.03 & 0.51 to 2.10 & 0.93 \\
\hline Acute infection & $11(0.8)$ & $8(0.5)$ & 1.16 & 0.41 to 3.26 & 0.78 \\
\hline Chronic infection & $19(1.3)$ & $19(1.2)$ & 1.04 & 0.43 to 2.51 & 0.93 \\
\hline Past infection & $57(2.4)$ & $38(3.9)$ & 1.54 & 0.88 to 2.68 & 0.12 \\
\hline
\end{tabular}

*All models were adjusted for gravidity, use of a net/ITN, severe anaemia at enrolment, fever at enrolment and socioeconomic status. IST, intermittent screening and treatment; ITN, insecticide-treated bed net; P., Plasmodium; PCD, passive case detection; Pf, Plasmodium falciparum; Pv, Plasmodium vivax; RDT, rapid diagnostic test. 
Table 3 Prevalence of RDT-positive malaria at ANC visits 2,3 and 4

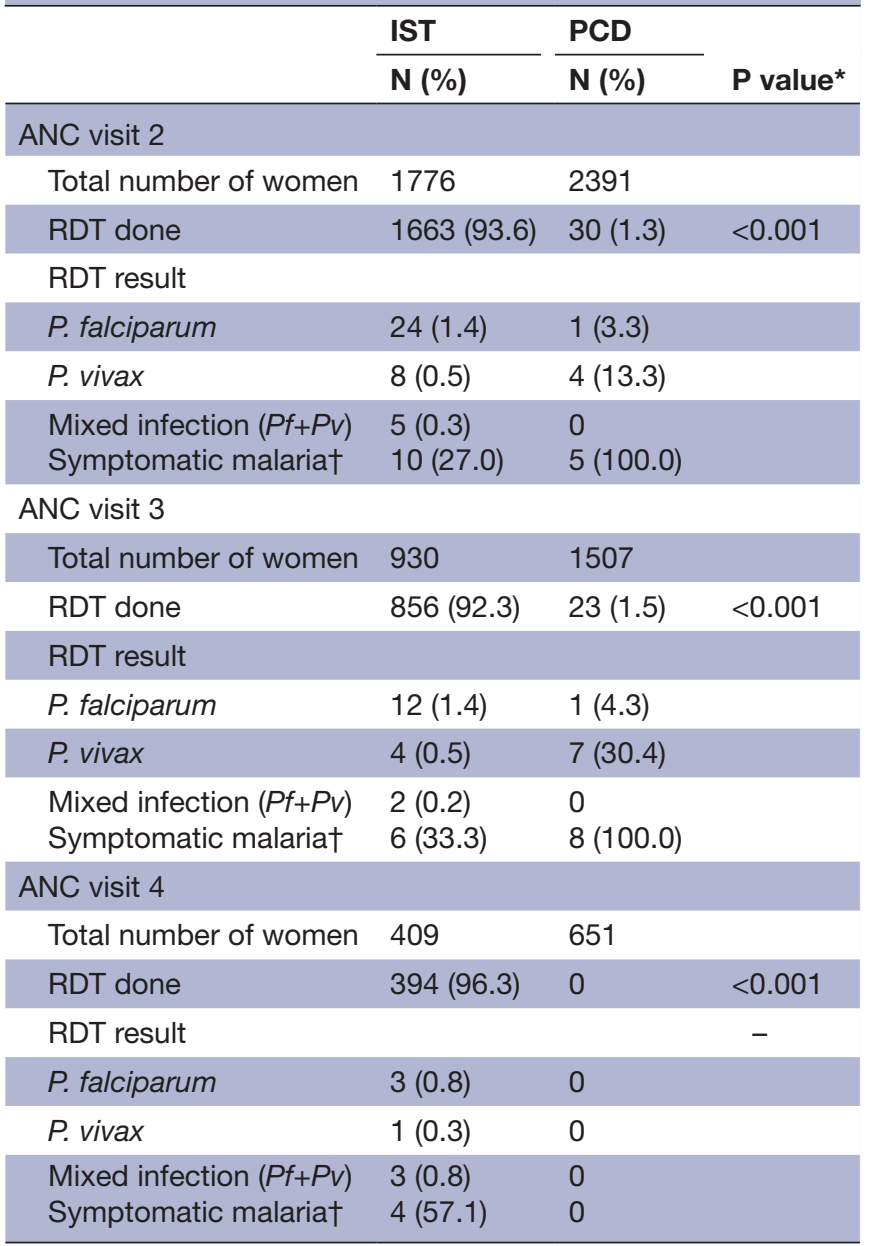

*Pearson's design-based test.

†RDT positive plus history of fever ( $<48$ hours) or temperature $>37.5^{\circ} \mathrm{C}$.

ANC, antenatal clinic; IST, intermittent screening and treatment; P., Plasmodium; PCD, passive case detection; Pf, Plasmodium falciparum; Pv, Plasmodium vivax; RDT, rapid diagnostic test.

was then divided into quintiles to create household socioeconomic groups. Baseline characteristics at enrolment were summarised and compared between the ISTp and PCD groups.

Pearson's design-based test was used to compare the prevalence of RDT, blood slide and PCR-positive malaria across study arms. To account for clustering by village in primary outcomes, a logistic random effects model was used to compare the odds of each outcome in the ISTp versus PCD groups. A quadrature check was applied to the model postestimation to assess the reliability of the model. An estimate of the between-cluster SD was obtained for each outcome from the models and assessed using a likelihood ratio test. Women's characteristics and measurements at enrolment were compared for women who had a biopsy and those who did not using Pearson's designbased test.

\section{Patient and public involvement statement}

The patients were not involved in the design, recruitment or conduct of the study. The results were disseminated to all relevant stakeholders, including the trial participants.

\section{RESULTS}

\section{Study population}

Between April 2012 and September 2014, we enrolled 3300 women in 46 clusters in the ISTp group and 3568 women in 41 clusters in the PCD group (figure 1). The total number of clusters and women in the two groups differed as some SHCs had to be closed due to security concerns. The cluster size ranged from 6 to 224 in the ISTp group and from 10 to 264 in the PCD group (table 1). The median size of the clusters was, however, similar in the two groups (93 and 92 women per cluster in ISTp and PCD, respectively). The proportion of women who attended ANC visits 2 and 3 was overall low $(61 \%$ and $35 \%)$ but even lower in the ISTp group than in the PCD group ( $54 \%$ vs $67 \%$ for visit $2(\mathrm{p}=0.01)$ and $28 \%$ vs $42 \%$ for visit $3(p=0.02)$ ) (figure 1$)$. However, the percentage of women who were seen at delivery was similar in each group (45\%). Almost all women were seen at the final 6-month postpartum home visit (99.8\%). The last delivery and postnatal follow-up took place in April and October 2015, respectively.

There were no significant differences in the distribution of age, gravidity, education, socioeconomic status and use of an ITN between the two trial groups (table 1). The proportion of women who reported a history of fever in the past 48 hours or temperature $>37.5^{\circ} \mathrm{C}$ was slightly higher at enrolment in the ISTp group $(8.5 \%$ vs $5.1 \%)$. The proportion of women who had received antimalarial treatment from the private sector at any time during their current pregnancy was high, $7.8 \%$ in the ISTp group and $7.1 \%$ in the PCD group.

\section{Prevalence of malaria at enrolment}

At enrolment, $95.8 \%$ of women in the IST group were tested with an RDT and $3.2 \%$ were malaria positive; $2.7 \%$ had a $P f, 0.3 \%$ a $P$. vivax $(P v)$ and $0.3 \%$ a mixed infection $(P f / P v)$ (table 1). According to national policy, RDT testing was restricted to those who had symptoms suggestive of malaria in the PCD group and only $3 \%$ of women had an RDT done at enrolment; of those $6.5 \%$ were tested positive for $P f, 2 \%$ for $P v$ and $0.9 \%$ for a mixed infection $(P f / P v)$. The proportion of women who were tested with PCR at enrolment was comparable between the groups (79.4\% vs $75.8 \%)$ and the prevalence of PCR positive malaria did not differ between the two groups $(5.9 \%$ vs $4.2 \%, \mathrm{p}=0.30$ ) (table 1). Reasons for women having no PCR result at enrolment include that the sample was either not collected or that the dried blood spots were too small for the amount needed for DNA extraction.

\section{Primary endpoint-placental malaria}

Placental biopsies were available from 1454 (44.0\%) women from the ISTp group and from 1560 (43.7\%) 
Table 4 Delivery and birth outcomes

\begin{tabular}{|c|c|c|c|c|c|}
\hline & \multirow{2}{*}{$\begin{array}{l}\text { IST } \\
\text { n/N (\%) }\end{array}$} & \multirow{2}{*}{$\begin{array}{l}\text { PCD } \\
\mathrm{n} / \mathrm{N}(\%)\end{array}$} & \multirow[b]{2}{*}{ Adjusted OR* } & \multirow[b]{2}{*}{$95 \% \mathrm{Cl}$} & \multirow[b]{2}{*}{ P value } \\
\hline & & & & & \\
\hline Total number of women & 3300 & 3568 & & & \\
\hline \multicolumn{6}{|l|}{ Birth weight $†$} \\
\hline$\geq 2.5 \mathrm{~kg}$ & $2590 / 2971$ (87.2) & $2771 / 3177$ (87.2) & & & \\
\hline$<2.5 \mathrm{~kg}$ & $381 / 2971$ (12.8) & $406 / 3177$ (12.8) & 1.07 & 0.86 to 1.34 & 0.56 \\
\hline \multicolumn{6}{|l|}{ Gestational age at birth $†$} \\
\hline Preterm $\ddagger$ & 515/3181 (16.2) & 645/3432 (18.8) & 1.31 & 0.81 to 2.10 & 0.27 \\
\hline Miscarriage§ or stillborn & 106/3240 (3.3) & $107 / 3452$ (3.1) & 1.12 & 0.72 to 1.74 & 0.62 \\
\hline Died $<24$ hours & $30 / 3131(0.96)$ & 28/3351 (0.84) & 1.44 & 0.45 to 4.62 & 0.54 \\
\hline Died $<1$ week & $49 / 3133(1.6)$ & $46 / 3406(1.4)$ & 0.96 & 0.51 to 1.81 & 0.89 \\
\hline \multicolumn{6}{|l|}{ Maternal Hb status $†$} \\
\hline $\mathrm{Hb} \geq 11 \mathrm{~g} / \mathrm{dL}$ & $186(12.6)$ & $236(14.7)$ & & & \\
\hline $\mathrm{Hb}<11 \mathrm{~g} / \mathrm{dL}$ & $1278(86.7)$ & $1347(84.1)$ & 1.17 & 0.83 to 3.91 & 0.14 \\
\hline $\mathrm{Hb}<7 \mathrm{~g} / \mathrm{dL}$ & $25(0.7)$ & $19(1.1)$ & 1.71 & 0.64 to 4.54 & 0.28 \\
\hline \multicolumn{6}{|l|}{ Maternal peripheral† blood } \\
\hline RDT positive & $34(2.4)$ & $25(1.6)$ & 1.30 & 0.67 to 2.52 & 0.44 \\
\hline PCR positive & $62(4.6)$ & $52(3.7)$ & 1.06 & 0.59 to 1.90 & 0.84 \\
\hline
\end{tabular}

*All models were adjusted for gravidity, use of a net/ITN, severe anaemia at enrolment, fever at enrolment and socioeconomic status.

†The total number of babies for each outcome varies due to missing values. $\mathrm{Hb}$ was only measured at delivery.

$\ddagger$ Gestational age $<37$ weeks.

$\S$ Spontaneous expulsion of fetus before 24 weeks of gestation.

$\mathrm{Hb}$, haemoglobin; IST, intermittent screening and treatment; ITN, insecticide-treated bed net; PCD, passive case detection; RDT, rapid diagnostic test.

from the PCD group. The proportion of women with active or past placental malaria was lower than expected in the study cohort overall $(5.0 \%, 152 / 3014)$. There was no statistically significant difference in the proportion of women with any placental malaria between the two groups $(6 \%$ vs $4.2 \%)$ or the proportion of women with active $(2.1 \%$ vs $1.7 \%)$ or past $(2.4 \%$ vs $3.9 \%)$ placental malaria (table 2). The ORs of active and past placental malaria in the IST group compared with the PCD group were $1.03(95 \%$ CI 0.51 to $2.10, p=0.93)$ and $1.54(95 \%$ CI 0.88 to $2.68, \mathrm{p}=0.12$ ), respectively. There were 87 cases of placental malaria in the IST group (table 2). However, only 10 of these cases had a positive RDT anytime during the ANC visits. Thus, the sensitivity of the RDT to detect placental malaria was $11.5 \%(10 / 87)$.

The risk of MiP varied substantially between clusters. The intracluster correlation coefficient for any placental malaria was 0.177 (95\% CI 0.066 to 0.395$)$. The number of placental malaria infections ranged from 0 to 9 per cluster; 29 clusters (14 ISTp and 15 PCD clusters) had no cases of placental malaria and five clusters had $>7$ cases of placental malaria (3 ISTp and 2 PCD clusters). However, the high and low placental malaria clusters were distributed equally between the two groups.

\section{Secondary endpoints}

The proportions of women in the ISTp group tested with an RDT during ANC visits 2, 3 and 4 were $1663 / 1776$ $(93.6 \%), 856 / 930 \quad(92.3 \%)$ and 394/409 (96.3\%), respectively, and among those tested, 2.2\%, $2.1 \%$ and $1.7 \%$, respectively, were positive for malaria (table 3). In the PCD group, the proportions of women suspected to have malaria and tested with an RDT at ANC visits 2 and 3 were $30 / 2391(1.3 \%)$ and 23/1507 (1.5\%), respectively, and among those tested, $16.7 \%$ and $34.8 \%$, respectively, were positive for malaria; no women had an RDT at the ANC visit 4.

The proportion of women who had RDT-positive malaria at least once during ANC and treated with AS+SP across all ANC visits was significantly higher in the ISTp arm 4.9\% (163/3300) than in the PCD arm 0.6\% $(23 / 3568)(\mathrm{p}<0.001)$.

Of all women tested RDT positive, 71\% (132/186) had Pf malaria, 19\% (35/186) Pv malaria and 10\% (19/186) a mixed infection $(P f / P v)$.

There was no statistically significant difference in low birth weight, preterm delivery, stillbirth or early neonatal death between the two groups (table 4 ).

\section{Safety endpoints}

There were 27 maternal deaths (death during pregnancy, delivery or within 42 days of delivery); 12 in the ISTp group and 15 in the PCD group (table 5). There were four maternal deaths attributable to malaria (two in each group) determined by verbal autopsy. However, there were no cases of severe malaria admitted to the study hospital. 
Table 5 Distribution of causes of maternal deaths in the two comparison groups

\begin{tabular}{|c|c|c|}
\hline & \multirow{2}{*}{$\begin{array}{l}\text { ISTp } \\
\text { N (\%) }\end{array}$} & \multirow{2}{*}{$\begin{array}{l}\text { PCD } \\
\mathrm{N}(\%)\end{array}$} \\
\hline & & \\
\hline Number of maternal deaths & 12 & 15 \\
\hline \multicolumn{3}{|l|}{ Causes of death } \\
\hline Malaria & $2(16.7)$ & $2(13.3)$ \\
\hline Pneumonia & $1(8.3)$ & $2(13.3)$ \\
\hline Anaemia & 0 & $2(13.3)$ \\
\hline HIV & $1(8.3)$ & $1(6.6)$ \\
\hline Pulmonary TB & $1(8.3)$ & 0 \\
\hline Sepsis & 0 & $1(6.6)$ \\
\hline Meningitis & $1(8.3)$ & 0 \\
\hline $\begin{array}{l}\text { Pregnancy-induced } \\
\text { hypertension/eclampsia }\end{array}$ & 0 & $4(26.6)$ \\
\hline Other cardiac diseases & 0 & $2(13.3)$ \\
\hline Epilepsy & $1(8.3)$ & 0 \\
\hline Cirrhosis & $1(8.3)$ & 0 \\
\hline Reproductive tract neoplasm & $1(8.3)$ & 0 \\
\hline Obstetric haemorrhage & $1(8.3)$ & $1(6.6)$ \\
\hline Suicide & $1(8.3)$ & 0 \\
\hline Undetermined & $1(8.3)$ & 0 \\
\hline
\end{tabular}

ISTp, intermittent screening and treatment during pregnancy; PCD, passive case detection; TB, tuberculosis.

\section{DISCUSSION}

ISTp detected more women with MiP compared with the current policy of PCD. However, ISTp did not reduce the risk of placental malaria or adverse birth outcomes compared with PCD. The possible reasons for ISTp not having an effect on placental malaria are: (i) only $28.2 \%$ of women were tested with an RDT at least three times during pregnancy in the IST group as they did not attend ANC frequently, (ii) the current generation of RDTs does not detect the majority of cases of placental malaria and (iii) a high proportion of women from both arms were treated with antimalarials from the private health sector. The most recent National Family Health Survey conducted in 2015-2016 reported that only $24.7 \%$ of mothers in rural Jharkhand had at least four ANC visits and $5.5 \%$ received full antenatal care, defined as mothers who had at least four ANC visits, one tetanus toxoid injection and at least 100 days of iron-folic acid tablets or syrup intake. ${ }^{15}$ The strengthening of ANC services would improve the effectiveness of interventions delivered as part of the existing ANC services and support the achievement of universal coverage. As part of ANC, a formal bed net distribution system for pregnant women and children would support the control of MiP. In our cohort, only $13.8 \%$ of pregnant women slept under an ITN the previous night.

The sensitivity of RDTs in pregnancy is lower than in non-pregnant populations. ${ }^{16}{ }^{17}$ In our study, the observed sensitivity of RDTs to detect histology-confirmed placental malaria was $11.5 \%$, similar to the sensitivity of $10 \%$ reported from Indonesia ${ }^{18}$ but lower to the sensitivity reported from Africa $(73 \%, 95 \%$ CI 64 to 81$) .{ }^{19}$

In low malaria transmission settings, where both symptomatic and asymptomatic patients should be identified and treated, the conventional RDTs miss important proportions of infections. New combination, highly sensitive RDTs able to identify all infected individuals, will support countries implementing active case detection strategies, ${ }^{20}$ but their use and added benefit for malaria case management in pregnancy will need to be assessed. ${ }^{21}$ The high maternal mortality ratio $(437 / 100$ $000)$ and perinatal mortality rate $(39.3 / 1000)$ observed in the study cohort suggest that the risk factors for adverse birth outcomes in this community are multifactorial, including a weak health system. Health systems challenges in Jharkhand also include insufficient access to care and potential treatment effects might be diluted by the high background rates of care seeking and treatment from the private sector. ${ }^{22} 23$

The prevalence of placental malaria in this study $(4.2 \%)$ was lower than expected but is comparable to the prevalence of placental parasitaemia detected by impression smear $(3.6 \%)$ in the study area in $2007 .{ }^{11}$ Placental biopsy samples were available from $<50 \%$ of the study participants because it was not feasible to collect placental samples when a woman delivered at home, in a private health facility, in a facility outside the study area or had an abortion. However, there was no difference in the baseline characteristics between the women with and without placental biopsy samples (online supplementary table 1). Thus, missing placental biopsy samples is unlikely to bias the comparison between the groups.

In Africa, the proportion of women detected to have malaria by routine screening is much higher on first attendance at an ANC than on subsequent visits, ${ }^{5}$ making single screening at first ANC attendance a potentially useful approach to malaria control. This was not the case in this study, where RDT-positive malaria ranged 2\%-3\% at all ANC visits. ISTp was shown to be inferior to IPTp with SP in several African settings. ${ }^{6}$ However, in areas with perennial transmission and SP resistance, in east Africa, it was shown that IPTp-SP was superior to ISTp in reducing adverse birth outcomes. ${ }^{24}$

A cross-sectional survey of children aged $<5$ years in the study area in 2014 showed a malaria prevalence of $5.2 \%$, $6.4 \%$ and $8.5 \%$ by blood slide, RDT and PCR, respectively. Even though malaria transmission is very low in the study setting, the majority of women with MiP were asymptomatic, suggesting that PCD does not appropriately control MiP. RDT-positive malaria ranged 2\%-3\% at all ANC visits in this low transmission setting and ISTp implemented through the routine ANC system was able to detect more malaria cases compared with PCD. PCD misses detection of a significant proportion of pregnant women carrying parasite and screening as part of ANC could be a useful approach to detect and treat MiP. However, with the current generation of RDTs and low 
attendance of repeat ANC visits, monthly ISTp is unlikely to have a major impact on the risk of placental malaria or adverse pregnancy outcomes relative to the existing strategy that relies largely on PCD.

This study has several limitations: (i) the prevalence of $\mathrm{MiP}$ was lower than expected, (ii) the cluster sizes varied considerably and (iii) a large proportion of placental samples could not be collected. Despite these limitations, the results allow to conclude that intermittent screening and treatment during ANC is a useful approach to detect and treat MiP. Any potential ISTp screening strategy in India would need to consider the individual risk of $P f$ and/or $P v$ infection. Screening of pregnant women at their first ANC visit and re-testing only positive cases at subsequent visits would be a possible approach. Women tested positive are also more likely to return for repeat ANC visits. The feasibility and cost implications, especially in combination with conventional or new highly sensitive RDTs, would, however, need to be assessed.

\section{Author affiliations}

${ }^{1}$ Department of Disease Control, London School of Hygiene \& Tropical Medicine Faculty of Infectious and Tropical Diseases, London, UK

${ }^{2}$ National Institute of Malaria Research, New Delhi, India

${ }^{3}$ Department of Pathology, Ispat General Hospital, Rourkela, India

${ }^{4}$ Office of Infectious Diseases, National Foundation for the Centers for Disease Control and Prevention Inc, Atlanta, Georgia, USA

${ }^{5}$ Department of Clinical Sciences, Liverpool School of Tropical Medicine, Liverpool, UK

Acknowledgements We would like to acknowledge the efforts of late Dr Bharatendu Shahi who was instrumental in setting up this trial. We also acknowledge the support and co-operation of the cardinal Telesphore Toppo and Ranchi Catholic Archdiocese. We thank Sr Jacintha Baxa and all the sisters of the St. Ursula mission hospital for all their support and efforts in the conduct and management of this trial. We thank Dr Celine Kujur for her continuous support in the initial stages of the trial, Sagya Singh (Malaria Control Programme Office, Ranchi), Dr Manoj Das (NIMR Field Unit, Ranchi), Dr K Pradhan (NIMR Field Unit, Rourkela), Varma (Mahadevi Birla Hospital, Ranchi), Dr Bara (DM0, Gumla), Dr Khanna (DMO Simdega) and the population of Basia, Kolibera, Simdega and Bano.

Contributors DC, JW, FtK and BG conceived the idea of the study. DC, JW, NV, AKA and NM initiated the study design. ISK, DC and VM helped with the implementation. ARM, SS and PB were doing the histopathological evaluation of the placental biopsies. JB provided statistical expertise in clinical trial design and was conducting the primary statistical analysis. All the authors contributed to the refinement of the study protocol and approved the final manuscript.

Funding The study was funded by the Malaria in Pregnancy Consortium, which was funded through a grant from the Bill \& Melinda Gates Foundation to the Liverpool School of Tropical Medicine.

Competing interests None declared.

Patient consent for publication Not required

Ethics approval Ethics Committee London School of Hygiene \& Tropical Medicine, 6017 Institutional Ethics Committee of the National Institute of Malaria Research, Delhi, India (approval was given without specific ID) and Government of India, Ministry of Tribal Affairs (approval was given without specific ID).

Provenance and peer review Not commissioned; externally peer reviewed.

Data availability statement Data are available on reasonable request.

Open access This is an open access article distributed in accordance with the Creative Commons Attribution Non Commercial (CC BY-NC 4.0) license, which permits others to distribute, remix, adapt, build upon this work non-commercially, and license their derivative works on different terms, provided the original work is properly cited, appropriate credit is given, any changes made indicated, and the use is non-commercial. See: http://creativecommons.org/licenses/by-nc/4.0/.

\section{REFERENCES}

1. Takem EN, D'Alessandro U. Malaria in pregnancy. Mediterr J Hematol Infect Dis 2013;5:e2013010.

2. Desai M, ter Kuile FO, Nosten F, et al. Epidemiology and burden of malaria in pregnancy. Lancet Infect Dis 2007;7:93-104.

3. WHO Recommendations (2015) Intermittent screening and treatment in pregnancy and the safety of ACTs in the first trimester Global Malaria Programme.

4. Chico RM, Cano J, Ariti C, et al. Influence of malaria transmission intensity and the $581 \mathrm{G}$ mutation on the efficacy of intermittent preventive treatment in pregnancy: systematic review and metaanalysis. Trop Med Int Health 2015;20:1621-33.

5. Tagbor H, Bruce J, Agbo M, et al. Intermittent screening and treatment versus intermittent preventive treatment of malaria in pregnancy: a randomised controlled non-inferiority trial. PLoS One 2010;5:e14425.

6. Desai M, Hill J, Fernandes S, et al. Prevention of malaria in pregnancy. Lancet Infect Dis 2018;18:e119-32.

7. Anvikar AR, Kuepfer I, Mishra V, et al. Efficacy of two artemisininbased combinations for the treatment of malaria in pregnancy in India: a randomized controlled trial. Malar J 2018;17:246.

8. Dellicour S, Tatem AJ, Guerra CA, et al. Quantifying the number of pregnancies at risk of malaria in 2007: a demographic study. PLoS Med 2010;7:e1000221.

9. Diamond-Smith N, Singh N, Gupta RKD, et al. Estimating the burden of malaria in pregnancy: a case study from rural Madhya Pradesh, India. Malar J 2009;8:24

10. India Go. Census India 2011.

11. Hamer DH, Singh MP, Wylie BJ, et al. Burden of malaria in pregnancy in Jharkhand State, India. Malar J 2009;8:210.

12. Nichols EK, Byass P, Chandramohan D, et al. The WHO 2016 verbal autopsy instrument: An international standard suitable for automated analysis by InterVA, InSilicoVA, and Tariff 2.0. PLoS Med 2018;15:e1002486.

13. Byass $P$, Chandramohan D, Clark SJ, et al. Strengthening standardised interpretation of verbal autopsy data: the new InterVA-4 tool. Glob Health Action 2012;5:19281-8.

14. Bulmer JN, Rasheed FN, Francis N, et al. Placental malaria. I. Pathological classification. Histopathology 1993;22:211-8.

15. International Institute for Population Sciences (IIPS) (2016-16) National Family Health Survey (NFHS-4), India, 2015-16: Jharkhand.

16. Kattenberg JH, Ochodo EA, Boer KR, et al. Systematic review and meta-analysis: rapid diagnostic tests versus placental histology, microscopy and PCR for malaria in pregnant women. Malar $J$ 2011;10:321.

17. Kattenberg JH, Tahita CM, Versteeg IAJ, et al. Evaluation of antigen detection tests, microscopy, and polymerase chain reaction for diagnosis of malaria in peripheral blood in asymptomatic pregnant women in Nanoro, Burkina Faso. Am J Trop Med Hyg 2012;87:251-6.

18. Ahmed R JRet al. Intermittent preventive treatment (IPT), intermittent screening and treatment (IST) and single screening and treatment (SST) with dihydroartemisinin-piperaquine for the control of malaria in pregnancy in Indonesia: A cluster-randomised, open-label superiority trial. Lancet Infect Dis 2019.

19. Williams JE, Cairns M, Njie F, et al. The Performance of a Rapid Diagnostic Test in Detecting Malaria Infection in Pregnant Women and the Impact of Missed Infections. Clin Infect Dis 2016;62:837-44.

20. Campillo A, Daily J, González IJ. International survey to identify diagnostic needs to support malaria elimination: guiding the development of combination highly sensitive rapid diagnostic tests. Malar J 2017;16:385

21. Vásquez AM, Medina AC, Tobón-Castaño A, et al. Performance of a highly sensitive rapid diagnostic test (HS-RDT) for detecting malaria in peripheral and placental blood samples from pregnant women in Colombia. PLoS One 2018;13:e0201769.

22. De Costa A, Johannson E. By 'default or design'? The expansion of the private health care sector in Madhya Pradesh, India. Health Policy 2011;103:283-9.

23. Witter S, Govender V, Ravindran TKS, et al. Minding the gaps: health financing, universal health coverage and gender. Health Policy Plan 2017;32(suppl 5):v4-12.

24. Desai M, Gutman J, L'lanziva A, et al. Intermittent screening and treatment or intermittent preventive treatment with dihydroartemisinin-piperaquine versus intermittent preventive treatment with sulfadoxine-pyrimethamine for the control of malaria during pregnancy in western Kenya: an open-label, three-group, randomised controlled superiority trial. Lancet 2015;386:2507-19. 